Special issue of the 3rd International Conference on Computational and Experimental Science and Engineering (ICCESEN 2016)

\title{
Monte Carlo Simulation Studies of Collimator Parameters for TARLA Bremsstrahlung Facility
}

\author{
Z.N. KuluÖZTÜRK ${ }^{a, *}$, N. Demir ${ }^{b}$ And İ. AKKurT ${ }^{c}$ \\ ${ }^{a}$ Bitlis Eren University, Vocational School of Health Services, Bitlis, Turkey \\ ${ }^{b}$ Uludağ University, Department of Physics, Bursa, Turkey \\ ${ }^{c}$ Süleyman Demirel University, Department of Physics, Isparta, Turkey
}

\begin{abstract}
In this work, calculations of the design of bremsstrahlung photon collimator within the scope of the Turkish Accelerator Center Project are presented. At TARLA facility (Turkish Accelerator Radiation Laboratory at Ankara), bremsstrahlung photons created by the accelerated electron beams, are transferred to the experimental area through the collimator, which has a conical geometry with length of $320 \mathrm{~cm}$. In this study, $\mathrm{Al}, \mathrm{Fe}$ and $\mathrm{Cu}$ materials were selected as collimator materials. All interactions between the collimator materials and Gaussian photon beam in $8-32 \mathrm{MeV}$ energy range $(8 \mathrm{MeV}, 16 \mathrm{MeV}, 24 \mathrm{MeV}$ and $32 \mathrm{MeV})$ were taken into account. The entry radius, geometry and chosen materials are important parameters for collimator design. The photon fluence from collimator, secondary particle distributions and the number of photons, scattered from the collimator, were calculated as functions of these parameters. All calculations were made with the Monte Carlo code FLUKA. According to the results of these simulations, collimator with conical geometry, made of aluminium, with $0.25 \mathrm{~cm}$ entry radius, was determined to be appropriate for TARLA bremsstarhlung photon facility of Turkish Accelerator Center.
\end{abstract}

DOI: 10.12693/APhysPolA.132.796

PACS/topics: 07.05.Tp, 02.70.Uu, 29.17.+w

\section{Introduction}

Collimation of a particle beam generated in an accelerator center is important in terms of beam characteristics and radiation safety in the area. At the same time, this process provides limits of the divergence of the transported beam. For this, at many accelerator centers, various collimator systems, fabricated with different geometries and materials are available [1-3]. The type and energy of collimated particles are effective factors for optimizing collimator design.

In this work, a useful collimation system for the bremsstrahlung photons of the TARLA facility was modelled using FLUKA code [4]. Simulation programming is indispensable tool in optimisation studies of such a complex system. Changes of the transmitted photon flux, depending on the collimator material and geometry, were examined.

Furthermore, the photons passing through the collimator interact with collimator material and create secondary particles. To reduce the background radiation pollution the production of secondary particles should be minimized. Therefore, the appropriate material selection is important. In the previous study, energy deposition and dose distribution for the cylindrical collimator design were calculated [5]. In this paper the detailed results of calculations for conical geometry are presented. It was observed that conical geometry is more efficient than the cylindrical one.

*corresponding author; e-mail: demircizehranur@gmail.com

\section{Materials and methods}

\subsection{Photon collimator design in FLUKA}

FLUKA is a Monte Carlo program developed to calculate particle transport and interaction of particles with matter. FLUKA can simulate with high accuracy the interaction with matter and propagation in matter of about 60 different particles. This simulation code uses an advanced version of well-known Combinatorial Geometry package. Therefore, it can even handle complex geometries [4]. In this study, the parameters of collimator which is one of the basic parts of TARLA bremsstrahlung photon facility have been determined using the FLUKA code.

\subsection{Collimator geometry}

The geometry of the collimator is one of the most important criteria for the transport of photons. Since the bremsstrahlung photons, which are formed after the interaction between the radiator and the accelerated electron beam, are spread in a conical shape; the collimator with conical geometry will be compatible with geometry of the photon beam and thus, more efficient.

For collimator with conical geometry, a cylinder with length of $320 \mathrm{~cm}$ and radius of $6 \mathrm{~cm}$ was selected. The interior of the cylinder was cut into a cone shape. The simulation studies were performed for four different values of the entrance radius of the cone, of $0.25,0.50$, 0.75 and $1.00 \mathrm{~cm}$. The exit radius of cone was chosen as $1.40 \mathrm{~cm}$. Vacuum was assumed to be inside the collimator.

In the FLUKA simulation calculations, the collimator with a length of $320 \mathrm{~cm}$ was divided into ten regions, in order to follow the secondary particles, which are formed in the collimator material. The length of each region 
is $32 \mathrm{~cm}$. When these $32 \mathrm{~cm}$ long regions were considered, the conical regions inside the collimator, assumed to contain vacuum, were removed. Only the parts where the collimator material was present were taken into account.

\subsection{Collimator materials}

The intensity of the photon beam, to be transferred to the experiment area, is closely related to the collimator material. As is well known, with the increase of the photon absorption coefficient of the material, the transmitted photon intensity exponentially decreases. Materials with high absorption coefficient are not appropriate for collimator system.

In addition, in terms of radiation safety, reactions $(\gamma, \mathrm{n})$ that may occur in the collimator, should be minimized as much, as possible. This situation is especially important at high energies. Therefore, the neutron pollution is kept to a minimum. Besides, the mechanical and chemical durability and cheapness of the used material are important factors in selecting the material. Aluminium, copper and iron metals were used for the simulations and the required properties of these metals are given in Table I.

\section{TABLE I}

Features of the collimator materials.

\begin{tabular}{c|c|c|c}
\hline \hline Features & $\mathrm{Al}$ & $\mathrm{Cu}$ & $\mathrm{Fe}$ \\
\hline Atom number $(\mathrm{Z})$ & 13 & 29 & 26 \\
Density $\left[\mathrm{g} / \mathrm{cm}^{3}\right]$ & 2.70 & 8.96 & 7.86 \\
Radiation length $\left(x_{0}\right)\left[\mathrm{cm}^{2}\right]$ & 24.26 & 13.16 & 14.14 \\
Melting point $\left[{ }^{\circ} \mathrm{C}\right]$ & 660.32 & 1084.62 & 1538 \\
Melting point $[\mathrm{K}]$ & 933.47 & 1357 & 1811 \\
Neutron separation energy, & 13.1 & 9.9 & 11.2 \\
$S_{n}[\mathrm{MeV}]$ & & &
\end{tabular}

\subsection{Photon fluence and secondary particle tracking}

In this study, the photon beam for simulations was defined by taking into account the distribution of bremsstrahlung photons, resulting from the interaction of the radiator with accelerated electrons, coming from linac-1 and linac-2. At the same time, bremsstrahlung photons with energy up to $80 \%$ of the electron energy, coming from the linacs can be obtained [6]. Thus, the photon beam entering the collimator was assumed to have 8,16 , 24 and $32 \mathrm{MeV}$ and to have Gaussian distribution. This Gauss beam is calculated based on the distribution of bremsstrahlung photons, produced from the thin tantalum radiator [7]. Calculations were made for four different energies $(8,16,24,32 \mathrm{MeV})$ and the program was run for three cycles, for $10^{6}$ photons.

The most important parameter that determines the optimal structure of the collimator is the photon fluence, carried through the collimator. The calculations were repeated for four different entrance radii $(0.25,0.50,0.75$ and $1.00 \mathrm{~cm}$ ) and exit radius of $1.40 \mathrm{~cm}$, and the variations of the photon numbers depending on the entrance radius were investigated. These values, calculated per photon, are given in Tables II-V.
TABLE II

Photon numbers from $\mathrm{Al}, \mathrm{Cu}$ and Fe collimators for photon beam with energy of $8 \mathrm{MeV}$.

\begin{tabular}{c|c|c|c|c}
\hline \hline Collim. & \multicolumn{4}{|c}{ Photon number } \\
\cline { 2 - 5 } Mater. & $0.25 \mathrm{~cm}$ & $0.50 \mathrm{~cm}$ & $0.75 \mathrm{~cm}$ & $1 \mathrm{~cm}$ \\
\hline $\mathrm{Al}$ & $2.19 \times 10^{-3}$ & $5.65 \times 10^{-3}$ & $8.69 \times 10^{-3}$ & $1.07 \times 10^{-2}$ \\
$\mathrm{Cu}$ & $1.65 \times 10^{-3}$ & $5.14 \times 10^{-3}$ & $8.34 \times 10^{-3}$ & $1.06 \times 10^{-2}$ \\
$\mathrm{Fe}$ & $1.67 \times 10^{-3}$ & $5.01 \times 10^{-3}$ & $8.38 \times 10^{-3}$ & $1.04 \times 10^{-2}$
\end{tabular}

TABLE III

Photon numbers from $\mathrm{Al}, \mathrm{Cu}$ and Fe collimators for photon beam with energy of $16 \mathrm{MeV}$.

\begin{tabular}{c|c|c|c|c}
\hline \hline Collim. & \multicolumn{4}{|c}{ Photon numbers } \\
\cline { 2 - 5 } Mater. & $0.25 \mathrm{~cm}$ & $0.50 \mathrm{~cm}$ & $0.75 \mathrm{~cm}$ & $1 \mathrm{~cm}$ \\
\hline $\mathrm{Al}$ & $9.06 \times 10^{-3}$ & $2.21 \times 10^{-2}$ & $3.44 \times 10^{-2}$ & $4.17 \times 10^{-2}$ \\
$\mathrm{Cu}$ & $6.35 \times 10^{-3}$ & $1.97 \times 10^{-2}$ & $3.27 \times 10^{-2}$ & $4.14 \times 10^{-2}$ \\
$\mathrm{Fe}$ & $6.41 \times 10^{-3}$ & $1.98 \times 10^{-2}$ & $3.28 \times 10^{-2}$ & $4.12 \times 10^{-2}$
\end{tabular}

TABLE IV

Photon numbers from $\mathrm{Al}, \mathrm{Cu}$ and $\mathrm{Fe}$ collimators for photon beam with energy of $24 \mathrm{MeV}$.

\begin{tabular}{c|c|c|c|c}
\hline \hline Collim. & \multicolumn{4}{|c}{ Photon numbers } \\
\cline { 2 - 5 } Mater. & $0.25 \mathrm{~cm}$ & $0.50 \mathrm{~cm}$ & $0.75 \mathrm{~cm}$ & $1 \mathrm{~cm}$ \\
\hline $\mathrm{Al}$ & $1.93 \times 10^{-2}$ & $4.76 \times 10^{-2}$ & $7.39 \times 10^{-2}$ & $9.00 \times 10^{-2}$ \\
$\mathrm{Cu}$ & $1.34 \times 10^{-2}$ & $4.23 \times 10^{-2}$ & $7.04 \times 10^{-2}$ & $8.90 \times 10^{-2}$ \\
$\mathrm{Fe}$ & $1.37 \times 10^{-2}$ & $4.26 \times 10^{-2}$ & $7.07 \times 10^{-2}$ & $8.90 \times 10^{-2}$
\end{tabular}

TABLE V

Photon numbers from $\mathrm{Al}, \mathrm{Cu}$ and Fe collimators for photon beam with energy of $32 \mathrm{MeV}$.

\begin{tabular}{c|c|c|c|c}
\hline \hline Collim. & \multicolumn{4}{|c}{ Photon numbers } \\
\cline { 2 - 5 } Mater. & $0.25 \mathrm{~cm}$ & $0.50 \mathrm{~cm}$ & $0.75 \mathrm{~cm}$ & $1 \mathrm{~cm}$ \\
\hline $\mathrm{Al}$ & $3.17 \times 10^{-2}$ & $7.94 \times 10^{-2}$ & 0.124 & 0.151 \\
$\mathrm{Cu}$ & $2.23 \times 10^{-2}$ & $7.10 \times 10^{-2}$ & 0.119 & 0.150 \\
$\mathrm{Fe}$ & $2.27 \times 10^{-2}$ & $7.12 \times 10^{-2}$ & 0.119 & 0.150
\end{tabular}

When Tables II-V are examined, the maximum number of photons is seen in $\mathrm{Al}$ collimator. As can be seen from the results, the difference between the number of photons from $\mathrm{Cu}$ and $\mathrm{Fe}$ collimators is insignificant. It has been observed that as the energy of bremsstrahlung photons entering the collimator increases, the number of photons coming out from the collimator also increases.

Two-dimensional representations of the photon fluence, obtained for considered collimators, are given in Figs. 1-6 for $16 \mathrm{MeV}$ and $32 \mathrm{MeV}$ photon beam. Figures $1-6$ are plotted using FLUKA, to compare the photon fluence formed by the smallest $(0.25 \mathrm{~cm})$ and the largest $(1 \mathrm{~cm})$ entrance radius of the collimator.

Figures 1-6 show that the highest photon flux is at the entrance of the collimator and the distribution of photons, due to interaction of photon beam with the collimator material, decreases towards the exit of the collimator. At the same time it is seen that the photon fluence decreases towards the outside of the collimator. 


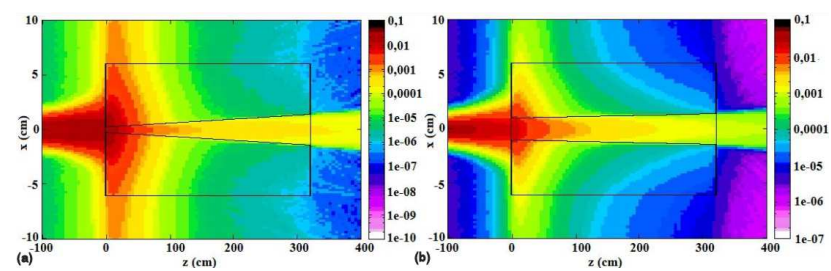

Fig. 1. Photon fluence (photon $/ \mathrm{cm}^{2} /$ primary photon) from $\mathrm{Al}$ collimator for photon energy of $16 \mathrm{MeV}$ and for entrance radius of (a) $0.25 \mathrm{~cm}$, (b) $1 \mathrm{~cm}$.

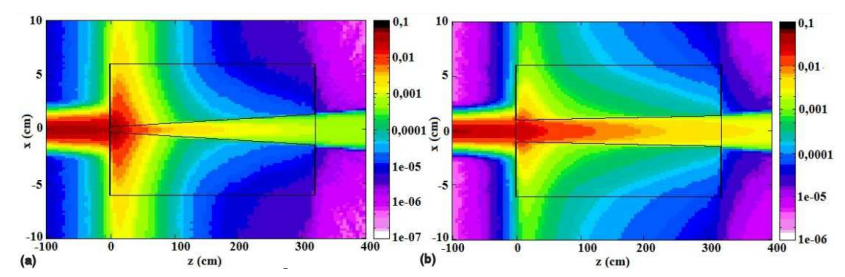

Fig. 2. Photon fluence (photon $/ \mathrm{cm}^{2} /$ primary photon) from $\mathrm{Al}$ collimator for photon energy of $32 \mathrm{MeV}$ and for entrance radius of (a) $0.25 \mathrm{~cm}$, (b) $1 \mathrm{~cm}$.

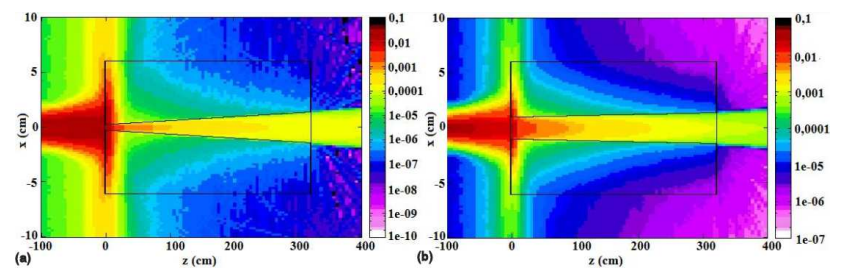

Fig. 3. Photon fluence (photon $/ \mathrm{cm}^{2} /$ primary photon) from $\mathrm{Cu}$ collimator for photon energy of $16 \mathrm{MeV}$ and for entrance radius of (a) $0.25 \mathrm{~cm}$, (b) $1 \mathrm{~cm}$.

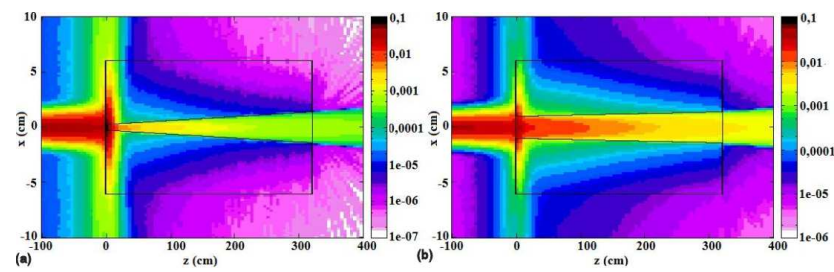

Fig. 4. Photon fluence (photon $/ \mathrm{cm}^{2} /$ primary photon) from $\mathrm{Cu}$ collimator for photon energy of $32 \mathrm{MeV}$ and for entrance radius of (a) $0.25 \mathrm{~cm}$, (b) $1 \mathrm{~cm}$.

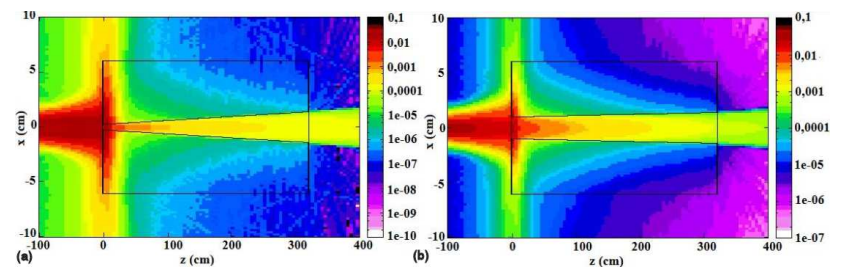

Fig. 5. Photon fluence (photon $/ \mathrm{cm}^{2} /$ primary photon) from Fe collimator for photon energy of $16 \mathrm{MeV}$ and for entrance radius of (a) $0.25 \mathrm{~cm}$, (b) $1 \mathrm{~cm}$.

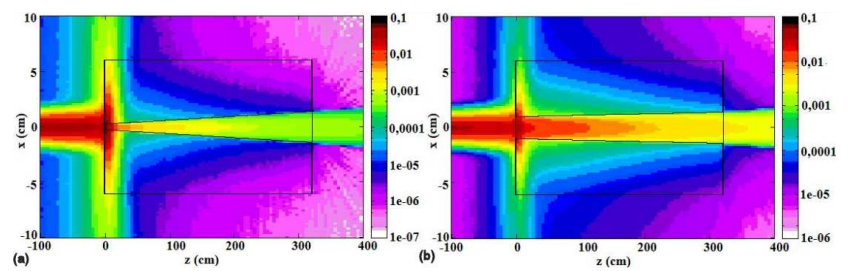

Fig. 6. Photon fluence (photon $/ \mathrm{cm}^{2} /$ primary photon) from Fe collimator for photon energy of $32 \mathrm{MeV}$ and for entrance radius of (a) $0.25 \mathrm{~cm}$, (b) $1 \mathrm{~cm}$.

As shown in Tables II-V, and it is seen in Figs. 1-6, that as the collimator entrance radius and the photon beam energy increase, the photon fluence from the collimator increases.

Determination of the entrance radius of the collimator geometry is important for two reasons, to determine the fluence of the photon beam to be used in the experiment area, and to determine the formation of secondary particles, that will affect the experimental area. Figures 7 and 8 , show changes in the direction of the $z$-axis of the distribution of electrons and neutrons, formed as a result of interaction with photon beam with energy of $16 \mathrm{MeV}$, for $\mathrm{Al}$ collimator with entrance radius of $0.25 \mathrm{~cm}$ and $1.00 \mathrm{~cm}$.
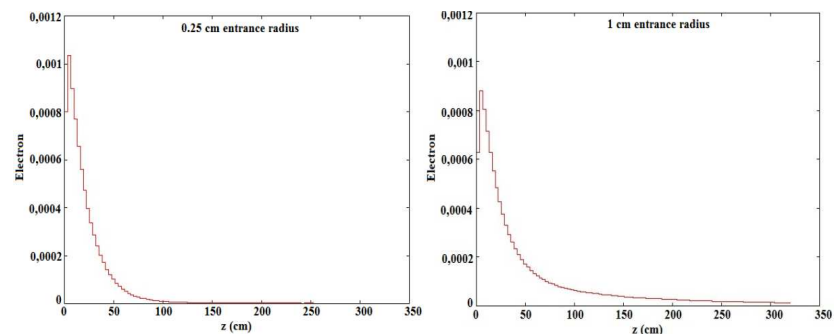

Fig. 7. Spectra of electrons formed in $\mathrm{Al}$ collimator for photon beam with energy of $16 \mathrm{MeV}$.
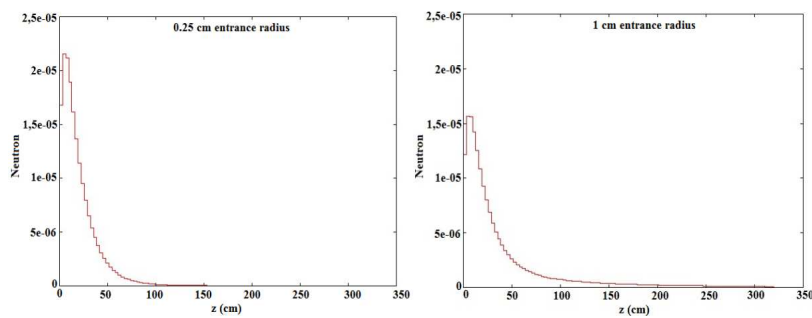

Fig. 8. Spectra of neutrons formed in $\mathrm{Al}$ collimator for photon beam with energy of $16 \mathrm{MeV}$.

In Figures 7-8 it is seen that the tail of the spectrum grows with the increasing opening radius. At the entrance of the collimator more particles are formed, as the interaction surface of the collimator with an entrance radius of $0.25 \mathrm{~cm}$ is larger.

In order to track the secondary particles, the collimator was divided into ten regions. The volume of each 
of these regions, in which the interior of the collimator was not included, was considered. The calculations were done for each region separately and the distributions of the secondary particles, formed by the bremsstrahlung photons along the path, were obtained based on the collimator material and the photon energy.

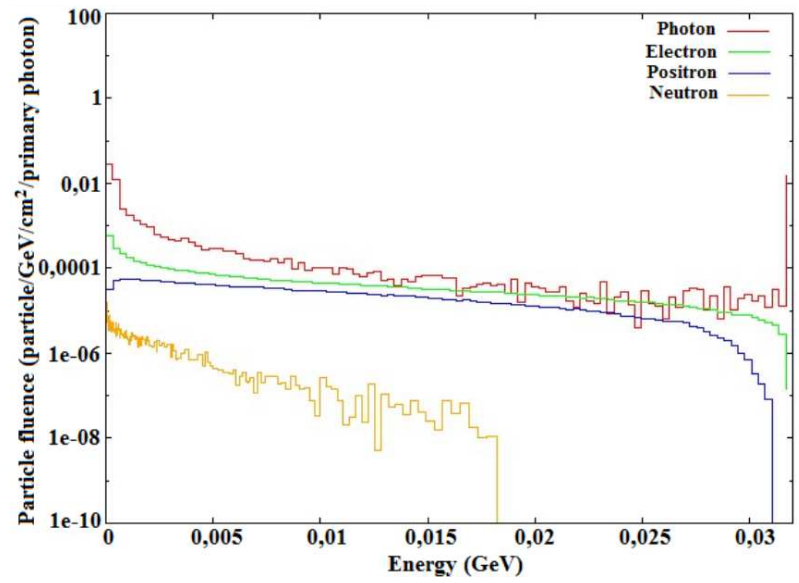

Fig. 9. Energy spectra of secondary particles, formed in $\mathrm{Al}$ collimator with radius of $0.25 \mathrm{~cm}$, for photon beam with energy of $32 \mathrm{MeV}$.

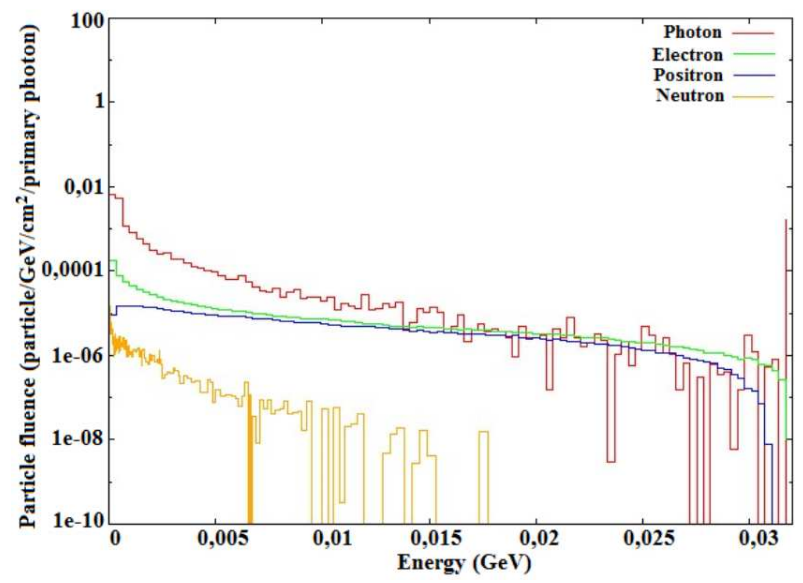

Fig. 10. Energy spectra of secondary particles, formed in $\mathrm{Cu}$ collimator with radius of $0.25 \mathrm{~cm}$, for photon beam with energy of $32 \mathrm{MeV}$.

In order to see the maximum density of secondary particles reaching the experimental area, the spectra of the secondaries in the last zone (10th region) of the $\mathrm{Al}, \mathrm{Cu}$ and Fe collimators are presented in Figs. 9-11.

\section{Conclusions}

In simulations of collimators, when photon fluences, obtained from $\mathrm{Al}, \mathrm{Cu}$ and Fe collimators, were compared, it was seen that the maximum photon fluences were obtained in Al collimator. Photon fluences from the collimator have increased with the increase of the energy of the incoming beam. The maximum photon fluencies were obtained from photon beam with energy of $32 \mathrm{MeV}$.

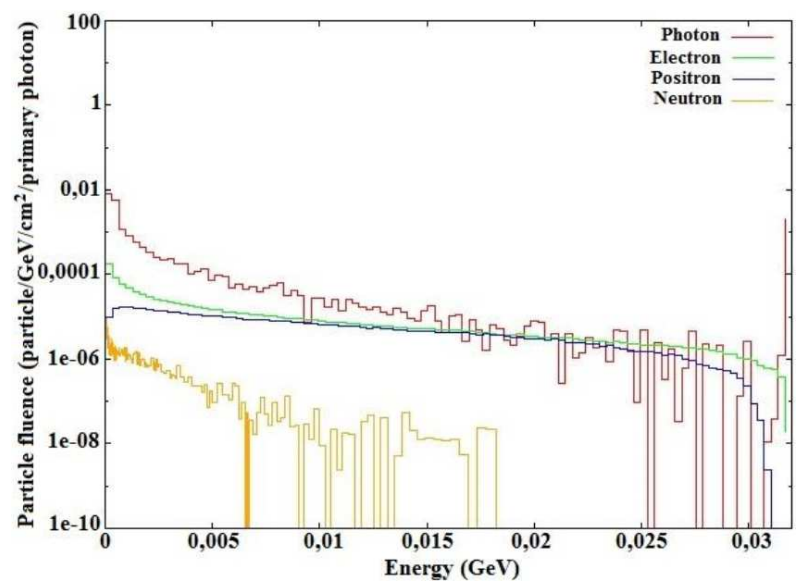

Fig. 11. Energy spectra of secondary particles, formed in Fe collimator with radius of $0.25 \mathrm{~cm}$, for photon beam with energy of $32 \mathrm{MeV}$.

In simulations of collimators with different entrance radii, it was determined that as the entrance radius of the collimator was increased, the photon fluence was also increased. However, when the secondary particles, formed in the collimator, are examined along the $z$-axis, it is seen that the number of these particles also increases with the radius. The geometry with the minimum number of secondary particles, scattered towards the experimental area, was determined to have the entrance radius of $0.25 \mathrm{~cm}$.

For radiation safety, neutron outputs, in particular, should be minimized as far as possible. Otherwise, a neutron shielding must be employed in the facility. On the basis of the obtained results, when $\mathrm{Al}, \mathrm{Cu}$ and $\mathrm{Fe}$ collimators are used, neutron outputs were found to be insignificant, especially for Al material.

Considering all simulation studies, the maximum photon flux was obtained in $\mathrm{Al}$ collimator and therefore $\mathrm{Al}$ was selected as the collimator material. It is determined that the entrance radius of the collimator of $0.25 \mathrm{~cm}$ is the most appropriate solution.

As a result of this study, collimator made of $\mathrm{Al}$ material with $0.25 \mathrm{~cm}$ entrance and $1.40 \mathrm{~cm}$ exit radius, with conical geometry and $320 \mathrm{~cm}$ length is suggested for collimator equipment of TARLA bremsstrahlung photon facility.

\section{Acknowledgments}

This work has been supported by State Planning Organization of Turkey under project number of DPT2006K120470 and Suleyman Demirel University Foundation Unit (BAP) with the project number 3407-D2-12.

\section{References}

[1] P. Mohr, J. Enders, T. Hartmann, H. Kaiser, D. Schiesser, S. Schmitt, S. Volz, F. Wissel, A. Zilges, Nucl. Instrum. Meth. A 423, 480 (1999). 
[2] R. Schwengner, R. Beyera, F. Dönau, E. Grosse, A. Hartmann, A.R. Junghans, S. Mallion, G. Rusev, K.D. Schilling, W. Schulze, A. Wagner, Nucl. Instrum. Meth. A 555, 211 (2005).

[3] W. Mondelaers, K. Van Laere, A. Goedefroot, K. Van den Bossche, Nucl. Instrum. Meth. A 368, 278 (1996).

[4] A. Ferrari, P.R. Sala, A. Fasso, J. Ranft, FLUKA: a multi-particle transport code, CERN 2005-10, Geneva 2005.
[5] N. Demir, Z.N. Kuluöztürk, İ. Akkurt, Acta Phys. Pol. A 128, B-400 (2015).

[6] E.B. Podgorsak, Radiatıon Oncology Physics: A Handbook for Teachers and Students, International Atomic Energy Agency, Vienna 2005.

[7] Z.N. Demirci, M.Sc. Thesis, Süleyman Demirel Üniversitesi, Isparta 2011. 